\title{
Investigation of sexually transmitted protozoan parasite Tritrichomonas foetus in cattle in Bangladesh
}

\author{
M. R. Islam, M. H. Talukder*, M. B. Hossain and M. A. Karim \\ Department of Parasitology, Bangladesh Agricultural University, Mymensingh-2202, Bangladesh \\ *E-mail: talukdermhasan@bau.edu.bd
}

\begin{abstract}
Bovine trichomoniasis is a venereal disease caused by the protozoa Tritrichomonas foetus. The aim of the study was to investigate the prevalence of $T$. foetus in cattle and associated risk factors. A total of 200 samples from 183 cows and 17 bulls were collected from Veterinary Teaching Hospital, Bangladesh Agricultural University; Mymensingh district and Regional station of Bangladesh Livestock Research Institute in Shahjadpur, Sirajgonj during the period from March 2015 to May 2016. Among 200 cattle, 65 were indigenous, 100 were Holstein cross and 35 were Shahiwal cross bred. The collected samples were vaginal and uterine washing, aborted foetus and semen. The samples were collected from vagina and uterus by flashing with phosphate buffer solution by artificial insemination tube connected with $20 \mathrm{ml}$ syringe. The samples were examined for $T$. foetus under microscope. The samples were examined on the same day of collection. Out of 200 samples examined, $3(1.5 \%)$ were found infected with $T$. foetus protozoa. Among these 3 positive cases of tritrichomoniasis, one was detected in aborted foetal materials and other two were detected in vaginal washings. We did not find any $T$. foetus in the semen samples from the bulls. Therefore, bulls represent zero prevalence of $T$. foetus. During the study period the examined animals were categorized into three age groups viz. 40, 60 and 100 cattle for below 3 years, over 3 years to below 6 years and over 6 years to ten years, respectively. The prevalence rate was found in adult cattle over 6 years (>6-10 years) was $2.00 \%$, over 3 years (>3-6 years) was $1.66 \%$ and below 3 years (<3 years) was $0 \%$. In the study, prevalence rate of cross bred animals were $2.22 \%$ and no infection was found in indigenous bred. So, this preliminary study indicates that a low prevalence rate of bovine trichomoniasis present in cattle in this study area. Further investigation using serological and molecular method will help to detect precisely the prevalence of this important sexually transmitted protozoan disease in cattle. To our best knowledge, this is the first study on the prevalence of bovine tritrichomoniasis in Bangladesh except a single clinical case reported in 1974. This study also indicate that bovine tritrichomoniasis has a minor role related to abortion of cattle in Bangladesh.
\end{abstract}

Keyword: Prevalence, Tritrichomonas foetus, Cattle, Bangladesh

\section{Introduction}

$T$. foetus is an obligatory protozoa of reproductive tract of cattle. The disease characterized primarily by early pregnancy loss and occasionally by abortion and pyometra. The causative agent, $T$. foetus is a flagellated protozoan parasite sexually transmitted among cattle from infected, asymptomatic bulls to heifers or cows at the time of coitus and vice versa and mechanical transmission by artificial insemination, instrument or by gynecological examination (BonDurant, 1997; Parsonson et al., 1974). Trichomoniasis has a worldwide distribution and is a major cause of infertility in naturally bred cattle in many countries. Infected cows and heifers exhibit vaginitis, endometritis, early abortion, transient and permanent infertility (Felleisen, 1999). Bulls carry the protozoa in small number in the preputial cavity with some concentration in the fornix and the glans penis, localizing in the secretions (smegma) of the epithelial lining of the penis, prepuce and distal portion of the urethra (Hammond and Bartlett, 1943). Infected bulls are often asymptomatic carriers of $T$. foetus. However, they are very efficient in transmitting the organism to a cow or heifer during coitus. There are no lesions of diagnostic significance in bulls and the parasite does not affect either semen quality or sexual behavior. In bulls, a scant purulent preputial discharge associated with small nodules on the preputial and penile membranes may occur within the first two weeks of infection. Nevertheless, chronically infected bulls usually develop no gross lesions and are often clinically asymptomatic, They remain asymptomatic carriers of infection for years and possibly for life (Felleisen, 1999). Older bulls tend to become permanent carriers of $T$. foetus, perhaps as a result of the development of epithelial crypts in the preputial cavity of older bulls. 
Tritrichomonosis is included on the list of diseases notifiable to the World Organization for Animal Health (OIE). In the past, tritrichomonosis has been among the most economically devastating reproductive diseases. Losses have come mainly from abortions although increased veterinary costs and culling costs also contribute. It causes substantial economic losses wherever natural breeding conditions exist. It is the female that suffers the consequences of infection. $T$. foetus can persist in endemic herds without detection for many years and have a substantial economic impact on a cattle operation due to three factors: (1) reduced calf crop due to early embryonic loss or abortion; (2) reduced weaning weight due to delayed conception and (3) culling and replacement of infected cattle. However, in the case of artificial insemination, while $T$. foetus is capable of surviving the process used to freeze semen after collection, it is usually killed by drying or high temperatures. A number of studies in Bangladesh indicated that abortion in cows is one of the reasons of production loss in dairy farming. Shamsuddin et al., (1988) also studied reproductive diseases in large government dairy farm and identified retained placenta, metritis, pyometra, endometritis, cervicitis, persistent corporalutea, cystic ovaries and nonfunctional ovaries. These result in considerable economic loss to the dairy industry. Since no legal treatment exists, preventive and control measures focus on testing and culling positive animals, administration of a killed vaccine, educating the cattle producers, veterinarians regarding risk assessment and herd biosecurity. Therefore, a major component of education is establishing the prevalence of trichomoniasis in a region to aid in risk assessment. The economic loss could be well determining factor for financial failure of a cattle producer. In our country, abortion and repeat breeding are the important cause of herd economic loss but there is no proper investigation for identification or diagnosis of the causal agent and the preventive measures for these diseases.

There are many pathogens associated with abortion in cattle. These pathogens are protozoa, bacteria, virus, fungus, chlamydia, rickettsia and others. Among the protozoa associated with abortions are Toxoplasma gondii, Neospora caninum, Sarcocystis spp. and T. foetus. Ashfia (2015) reported that overall seroprevalence of $N$. caninum in cattle was found $1.81 \%$ in Bangladesh. Different studies across the world in dairy cattle showed seroprevalence ranging from $3 \%$ to $60 \%$ ( Talukder et al., 2016). The seroprevalence of T. gondii in cattle has been reported 12\% in Bangladesh (Shahiduzzaman et al., 2011 and Nahar, 2015). Among all the pathogens those are associated with abortion, some of these had been investigated in different times. To our best knowledge, there is no published article on the prevalence of $T$. foetus except a single clinical case reported in 1974 . Therefore, this study was aimed to investigate the prevalence and other associated risk factors of bovine trichomoniasis in the selected areas of Bangladesh.

\section{Materials and Methods}

\section{Study area}

Samples were collected from Veterinary Teaching Hospital, Bangladesh Agricultural University (BAU); Mymensingh district and Regional Station of Bangladesh Livestock Research Institute (BLRI) in Shahjadpur, Sirajgonj for the convenience of the study. Samples were collected from risk group of animals having history of abortion and reproductive infections. The collected samples were examined in the Department of Parasitology, BAU, Mymensingh and Animal Health Laboratory, BLRI, Sirajgonj for detecting the prevalence of trichomoniasis in cattle.

\section{Study period}

The research activities were carried out during the period from March, 2015 to May, 2016.

\section{Examination of cattle}

Total 200 samples were collected among which 135 from BLRI, 50 from Mymensingh district and 15 from Veterinary Teaching Hospital, BAU. Before sample collection age, sex, breed of animals and previous history of early abortion, delayed conception, pyometra, vaginitis and infertility were noted by interrogating the farmers. Out of 200 samples, 65 were indigenous bred, 100 were Holstein cross bred and 35 were Shahiwal cross bred and the samples were categorized into three age groups viz. below three years $(<3$ years), over three to six years (>3-6 years) and over six to ten years old ( $>6-10$ years). 


\section{Collection of sample}

Bulls: Total 17 semen samples were collected in Mymensingh district. The semen samples were collected from bulls during regular collection of their semen in Al station.

Cows: Total 183 samples were collected in which 135 from BLRI, 15 from Veterinary Teaching Hospital and 33 from Mymensingh district. After restraining the cow, swaps or washing with PBS from vagina and uterus were collected by artificial insemination pipette connected with $20 \mathrm{~mL}$ syringe managing all the hygienic conditions. In case of aborted foetal material, allantoic fluid, placenta, foetal membrane, foetal tissue etc. were collected. Out of 183 samples indigenous bred, Holstein cross bred and Shahiwal bred were 48, 100 and 35, respectively.

Sample analysis: The collected samples were examined under microscope for detection of $T$. foetus . First focused on low power objective (10x) and then at 40x magnification for identification of $T$. foetus (Figure 4). The samples were examined on the day of collection. The samples were examined repeatedly to find out the $T$. foetus.

Identification of the protozoa: $T$. foetus was identified on the basis of its characteristic morphological features. $T$. foetus is a flagellate, pyriform protozoa parasite, approximately 8-18 $\mu \mathrm{m}$ long and 4-9 $\mu \mathrm{m}$ wide with three anterior and one posterior flagella and an undulating membrane. The organisms move with a jerky, rolling motion and are seen in culture tests of preputial samples of infected bulls and vaginal washings or cervico-vaginal mucus of infected cows, or sometimes in aborted fetuses (Warton \& Honigberg, 1979).

\section{Statistical analysis}

Obtained data were compiled and analyzed by using Microsoft Excel.

\section{Results and Discussion}

\section{Overall prevalence of $T$. foetus infection in cattle}

In the present study, a total of 200 samples from cattle were examined in which 3 samples were found positive for $T$. foetus infection that indicates an overall prevalence 1.5\% (3/200) (Table 1 \& Fig. 1).

\section{Table 1. Prevalence rate of $T$. foetus infection in cattle}

The collected samples are categorized according to the infection:

\begin{tabular}{lcccc}
\hline Disease condition & No. of samples & Positive cases & Prevalence rate \\
\hline Repeat breeding/Infertility & 123 & 1 & $0.813 \%$ \\
Early abortion & 5 & 1 & $20 \%$ \\
Uterine Infection & 43 & & $0 \%$ \\
Pyometra/Endometritis/Metritis & 12 & 1 & $8.33 \%$ \\
Suspected Bulls & 17 & 0 & $0 \%$ \\
\hline Total & 200 & 3 & $1.5 \%$ \\
\hline
\end{tabular}

T. foetus is a pyriform shaped protozoa bearing three anterior flagella and one posterior flagellum and an undulating membrane (Fig. 4). 
From the result of present study, it was observed that bovine trichomoniasis was prevalent in the research area and 1.5\% cattle were infected with $T$. foetus. In Bangladesh, Rahman (1974) found only one clinical case of $T$. foetus infection in cattle. Parek et al., (1994) reported $28.61 \%$ cattle infected with $T$. foetus at Rajasthan in India. De et al., (1982) reported only one case of bovine trichomoniasis at the rural area of West Bengal in India. The other neighbouring countries such as Nepal, Bhutan and Myanmar where the disease was not reported till 2004 (OIE Handistatus, 2005). Yung et al., (2012) reported 5\% positive case in China and the authors reported that the infection also present in Asia, Australia, South America and South Africa. Joseph and Sivanandan (1987) also reported that they found 9 positive cases in two herds of Malaysia. These variation of infection might be due to differences in research areas, their geo-climatic conditions, methods of study, breeds of animals, sample size and types of services (Natural or $\mathrm{Al}$ ) present in that areas compared to the findings of this study. In the other studies, PCR had been done and it confirmed the infections but this study only based on the microscopic examination. Samples were based on the clinical symptoms and previous breeding histories of the cattle. Besides this, Bangladesh is a developing country where cattle rearing system is scavenging and semi-scavenging in most of it's parts. In these types of rearing, cattle graze in the field. So, the chance of natural mating is high. As a result, chance of infection is also high because the disease spread through coitus. In the rural areas, people normally serve their cow with natural mating. The infection is undiagnosed in most parts of Bangladesh due to lack of investigation throughout the country.

\section{Overall prevalence}

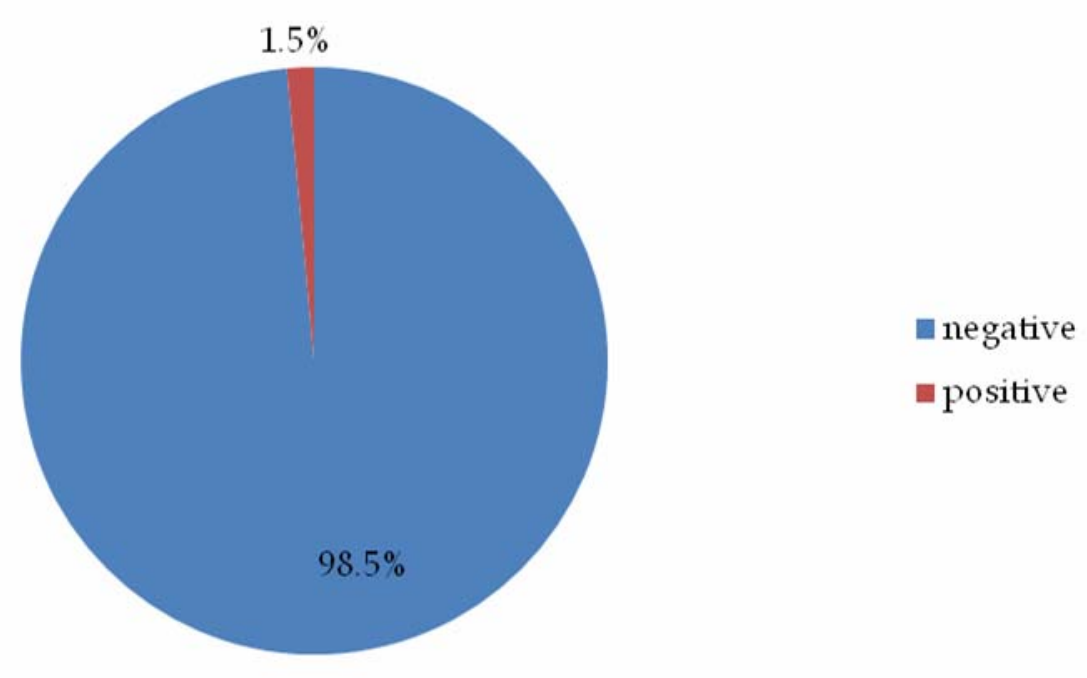

Fig. 1. Overall prevalence of $T$. foetus in cattle

\section{Age related prevalence of $T$. foetus infection in cattle}

No infection were detected among the young animals ( $<3$ years). However, $1.66 \%$ and $2.0 \%$ infection due to $T$. foetus were detected among the adult (>3-6 years) and aged animals (>6-10 years), respectively (Fig. 2).

It was revealed that age of the cattle had a significant effect on $T$. foetus infection. Among the age groups, prevalence rate of $T$. foetus infection in older cattle (>6-10 years) were $2.0 \%$, adult cattle ( $>3-6$ years) were $1.66 \%$ and no infection was found in young cattle ( $<3$ years) in this study. There is very few literature available focusing on such types of study. However, McCool et al., (1988) reported that the 
infection rate for trichomoniasis tended to increase with age, with a 30\% infection rate in animals of 10 years or older. Skirrow et al., (1985) reported that the age specific infection rates in a beef herd were $21.7 \%, 34.1 \%$ and $43.4 \%$ for the bulls of 3,4 and over 4 years old, respectively. Other authors, Clark et al., (1974) found higher rate of infection in older cattle by inoculating $T$. foetus directly into the posterior portion of the preputium. Clark and Colleagues found that only three of 19 bulls at 1-2 years old were infected, in contrast to 12 of 13 bulls at 3-7 years old (Clark et al., 1974). The same authors further found that all bulls older than 4 years were infected after three to six natural services, whereas only one of the two bulls of 3 years old was infected after nine services in a field study. They concluded that young (3 years) bulls were not as susceptible as older bulls in natural service. Laddas et al., (1973) conduct a survey of bulls in coastal and western Queensland and the Northern Territory of Australia, infection rates in young ( 9 months to 3 years), mature (3.5 to 7 years) and older bulls (7 years) were $0 \%, 25 \%$ and $37.2 \%$, respectively.

\section{Sex related prevalence of $T$. foetus infection in cattle}

Tritrichomoniasis was found only in $3(1.64 \%)$ cows and none of the bulls were infected among the examined animals (Fig. 3).

From the study, it was found that the prevalence rate of trichomoniasis in cows was $1.64 \%$. None of the bulls were infected with trichomoniasis in the study area. In this study, semen samples were collected from only 17 bulls whereas, 183 samples collected from different cows. Besides, these semen samples collected from only Al station not from free ranging bulls. This may be the possible cause of no infection was detected in the examined bulls.

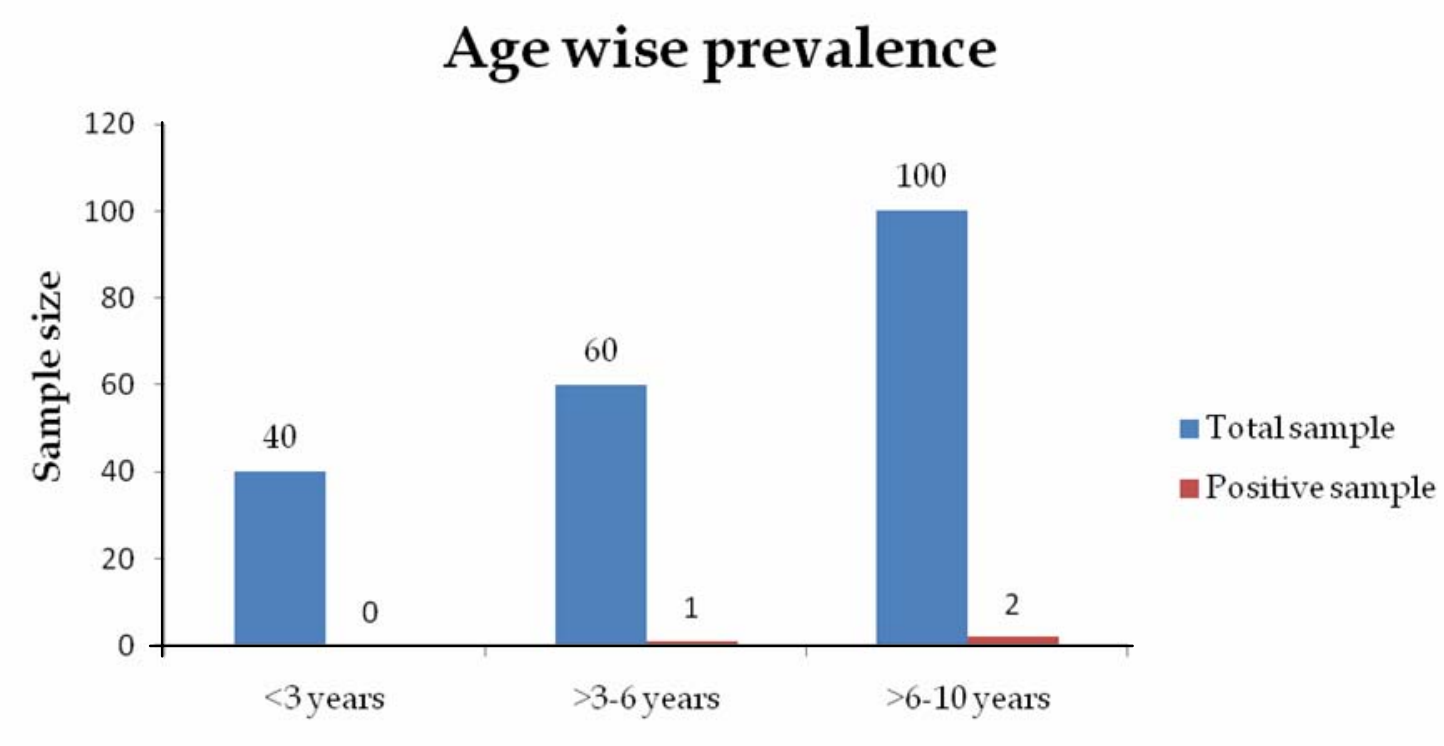

Age

Fig. 2. Age related prevalence of $T$. foetus in cattle 


\section{Sex wise prevalence}

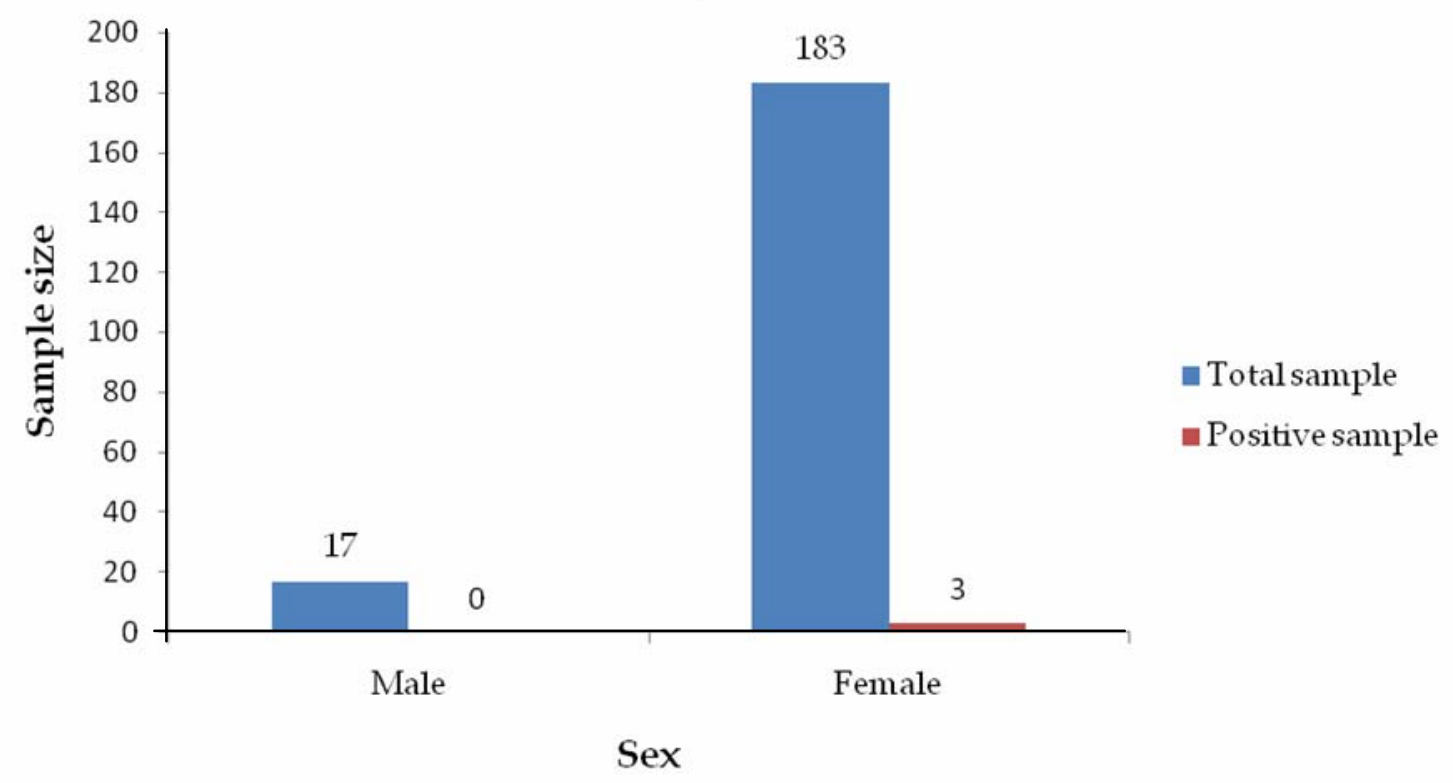

Fig. 3. Sex related prevalence of $T$. foetus in cattle.

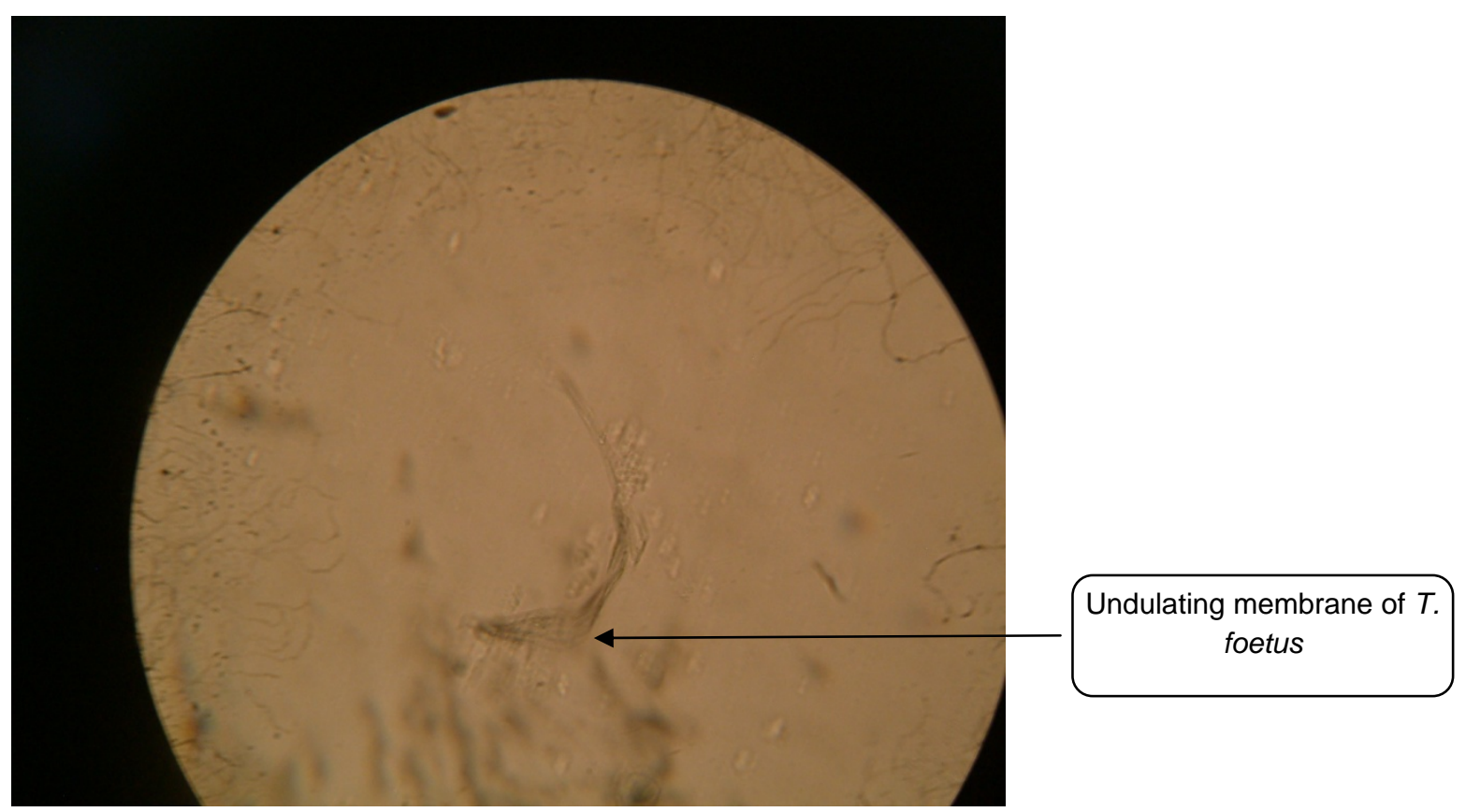

Fig. 4. Tritrichomonas foetus in cattle 


\section{Breed related prevalence of $T$. foetus infection in cattle}

Among the cross bred animals the prevalence of tritrichomoniasis was $2.22 \%$, in which Holstein cross bred was $1.48 \%$ and Shahiwal cross bred was $0.74 \%$. Whereas, no tritrichomoniasis was detected among indigenous cattle in this study.

From the study, it was found that the prevalence rate of trichomoniasis in cross bred cattle was $2.22 \%$, in which Holstein cross bred was $1.48 \%$ and Shahiwal cross bred was $0.74 \%$ whereas, no infection was found in indigenous cattle. As the cross breds are highly proliferative, give birth 12-13 calves and have to be inseminated more times. So, the chance of infection is also high compared to the indigenous cattle.

\section{Conclusion}

It has serious economic impact on the livestock production. This preliminary study indicates that a low prevalence rate of bovine trichomoniasis present in cattle in this study area. Further investigation using serological and molecular method will help to precisely detect the prevalence of this important sexually transmitted protozoan disease in cattle. This study also indicates that bovine trichomoniasis has a minor role related to abortion of cattle in Bangladesh.

\section{Acknowledgement}

The author gratefully acknowledge the financial support for this work from the Ministry of Science and Technology, Govt. of the peoples of Bangladesh through NST Fellowship (to Md Rafiul Islam).

\section{References}

Ashfia, K.M. 2015. Prevalence of Neospora caninum infection in cattle in the selected district of Bangladesh. MS Thesis. Department of Parasitology, Bangladesh Agricultural University, Mymensingh.

BonDurant, R.H. 1997. Pathogenesis, diagnosis and management of trichomoniasis in cattle. Veterinary Clinics of North America: Food Animal Practice, 13: 345-361.

Clark, B.L., Parsonson, I.M. and Dufty, J.H. 1974. Experimental infection of bulls with Tritrichomonas foetus. Australian Veterinary Journal, 50: 189-191.

De, B.N., Chatterjee, A., Bidyanta, J., Chakroborty, M., Deb, S.K., Mondal, P. and Sen, G.P. 1982. Note on the problems of breeding cows with special reference to common coital infections. Indian Journal of Animal Sciences, 52: 700-702.

Felleisen, R.S. 1999. Host-parasite interaction in bovine infection with Tritrichomonas foetus. Microbes Infection, 1: 807-816.

Hammond, D.M. and Bartlett, D.E. 1943a. Establishment of infection with Trichomonas foetus in bulls by experimental exposure. American Journal Veterinary Research, 4: 61-65.

Joseph, P.G. and Sivanandan, S.P. 1987. A report of the first isolation of Tritrichomonas foetus from bulls in Malaysia. Tropical Biomedicine, 4(2): 155-160.

Ladds, P.W., Dennett, D.P. and Glazebrook, J.S. 1973. A survey of the genitalia of bulls in Northern Australia. Australian Veterinary Journal, 49: 335-340.

McCool, C.J, Townsend, M.P., Wolfe, S.G., Simpson, M.A., Olm, T.C., Jayawardhana, G.A. and Carney, J.V. 1988. Prevalence of bovine venereal disease in the Victoria River District of the Northern Territory: likely economic effects and practicable control measures. Australian Veterinary Journal, 65: 153-156.

Nahar, F.H. 2015. Prevalence of Toxoplasma gondii infection in ruminants in selected herd in Bangladesh. MS Thesis. Department of Parasitology, Bangladesh Agricultural University, Mymensingh.

OIE Handistatus 2005. World Animal Health Publication and Handistatus II (data set for 2004). Paris, France: Office International des Epizooties.

Parek, P.K., Sharma, S.S., Bishnoi, B.L. and Gupta, A.K. 1994. Trichomoniasis in cattle in North West Rajasthan. Indian Journal of Animal Reproduction, 15(1): 27-28.

Parsonson, I.M., Clark, B.L. and Dufty, J.H. 1974. The pathogenesis of Tritrichomonas foetus infection of the bull. Australian Veterinary Journal, 50: 421-423.

Rahman, A. 1974. Bovine trichomoniasis in Bangladesh. Tropical Animal Health and Production, 6(2): 110. 
Shahiduzzaman, M., Islam, R., Khatun, M.M., Batanova, T.A., Kitoh, K. and Takashima, Y. 2011. Toxoplasma gondii seroprevalence in domestic animals and humans in Mymensingh District, Bangladesh. Journal of Veterinary Medical Science, 73(10): 1375-6

Shamsuddin, M., Alam, M.G.S. and Ahmed, J.U. 1988. Reproductive disorder of cross breed cows. Bangladesh Veterinary Journal, 22(24): 21-28.

Skirrow, S., BonDurant, R., Farley, J. and Correa, J. 1985. Efficacy of ipronidazole against trichomoniasis in beef bulls. Journal of the American Veterinary Medical Association, 187: 405-407.

Talukder, M.H., Ashfia, K.H. and Sah, R.P. 2016. Impact of Neosporosis in Dairy Cattle: Global and Bangladesh Perspectives. Proceedings of the $3^{\text {rd }}$ International Exhibition on Dairy, Aqua \& Pet, 3: 128-137.

Warton, A. and Honigberg, B.M. 1979. Structure of trichomonads as revealed by scanning electron microscopy. Journal of Protozoology, 26: 56-62.

Yang, N., Cui, X., Qian, W., Yu, S. and Liu, Q. 2012. Survey of nine abortifacient infectious agents in aborted bovine fetuses from dairy farms in Beijing, China, by PCR. Acta Veterinaria Hungarica, 60: 83-92. 